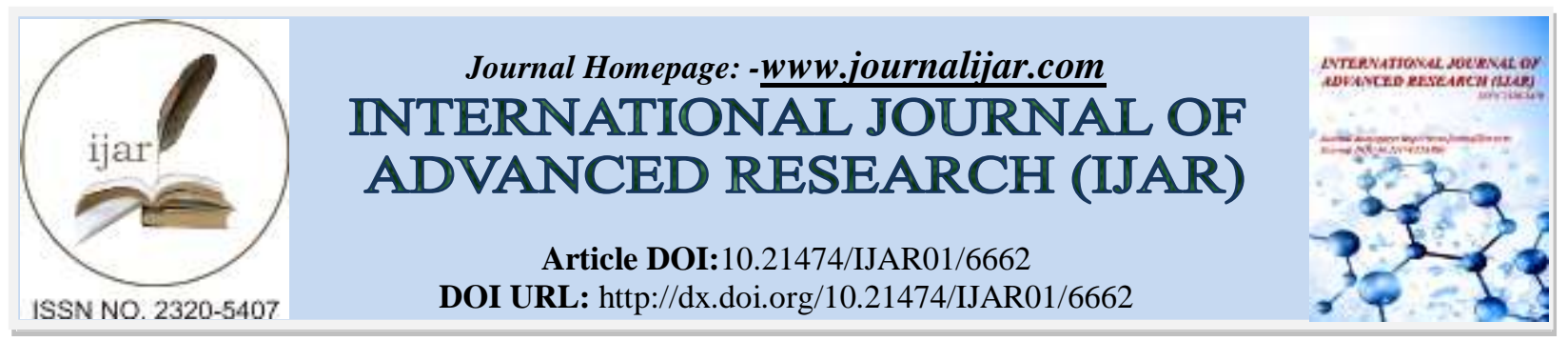

RESEARCH ARTICLE

\title{
JAUNDICE IN PREGNANCY: A 5 YEAR RETROSPECTIVE CROSS-SECTIONAL STUDY IN A TERTIARY CARE CENTRE.
}

\author{
Dr. Soumya Patil ${ }^{1}$, Dr. Kamal P. Patil ${ }^{2}$ and Dr. Anwar Mujawar ${ }^{3}$. \\ 1. Senior Resident, Department of Obstetrics and Gynecology, JNMC, Belgaum, India. \\ 2. Professor, Department of Obstetrics and Gynecology, JNMC, Belgaum, India. \\ 3. Associate Professor, Department of General medicine, JNMC, Belgaum, India.
}

\section{Manuscript Info}

\section{Manuscript History}

Received: 04 January 2018

Final Accepted: 06 February 2018

Published: March 2018

Keywords:-

Jaundice, HELLP, Hepatitis, Liver

disorder in Pregnancy

\begin{abstract}
Objective: To study the incidence, maternal and perinatal outcome in women presenting with jaundice in pregnancy.

Methods: This retrospective cross-sectional study was conducted in a tertiary care centre, catering to all sections of society. Women who presented with Jaundice in pregnancy over the past 5 years (September 2011 to August 2016) were retrospectively analyzed. Details regarding the maternal, obstetric and perinatal outcome were collected from the data in the medical records section.

Results: Out of 26,973 deliveries over the 5 year period, 123 cases of jaundice in pregnancy were reported. Incidence of jaundice in pregnancy in present study was found to be $0.45 \%$. Out of 123 women, $77(62.6 \%)$ had spontaneous onset of labour and $74(60 \%)$ delivered vaginally. $69.1 \%$ of them recovered, however $14.6 \%$ (18) needed ventilator support. HELLP syndrome (39.84\%) \& Viral hepatitis $(31.7 \%)$ were major causes of jaundice in present study. Maternal mortality rate was as high as $21 \%$. DIC and sepsis were leading causes of maternal mortality. Neonatal complications were reported in $47.9 \%$. Perinatal mortality was $30.8 \%$.

Conclusion: Jaundice in pregnancy results in high rates of maternal as well as fetal/neonatal morbidities and mortality. Early detection and timely initiation of proper management is essential to reduce the complications.

Copy Right, IJAR, 2018,. All rights reserved.
\end{abstract}

\section{Introduction:-}

Jaundice is a crucial sign during clinical examination suggesting either a pre-hepatic, hepatic or post-hepatic condition. Jaundice in pregnancy is regarded as high risk due to its high rates of morbidity and mortality. ${ }^{1}$ Incidence of jaundice in pregnancy is as high as 3-20\% in developing countries where as it is as low as $0.1 \%$ in developed countries. $^{2}$ In India, incidence is reported as 0.3 to $0.7 \% .^{3,4,5}$ Jaundice in pregnancy is largely categorized by 2 methods; one method classifies jaundice by taking into account its association with Pre-eclampsia (PE). Causes related to PE are predominantly HELLP (hemolysis, low platelet count and elevated liver enzymes) syndrome, AFLP (acute fatty liver of pregnancy) and PE itself where as hyperemesis and Intrahepatic cholestasis of pregnancy are unrelated to PE.1 An added method of classification categorizes liver diseases in pregnancy as intercurrent to pregnancy which includes viral hepatitis, drug induced, gall stones and second group as peculiar to pregnancy 
including cholestatic jaundice, AFLP, PE and HELLP syndrome and the last group as underlying chronic liver disease including cirrhosis of liver and chronic hepatitis. ${ }^{4,5}$

HELLP syndrome is a grievous complication in pregnancy characterized by hemolysis, elevated liver enzymes and low platelet count. It is an alarming complication of severe pre-eclampsia. This multisystem disorder complicates pregnancy in third trimester and it is the most common form of jaundice encountered in pregnancy due to the rise in the incidence of severe pre-eclampsia. It is associated with life threatening complications such as DIC, abruptio placenta, pulmonary edema, ARF, ARDS, stroke, hepatic failure and many more. ${ }^{4}$ Maternal mortality reported in this condition is $1 \%$. Management of HELLP syndrome demands multidisciplinary approach and referral to higher centre with facilities of blood and blood products. In order to reduce the risk of potentially serious complications, the only definitive treatment is immediate termination of pregnancy after stabilization of mother's condition. Other rare but serious conditions that mimic HELLP syndrome and pose difficulty in diagnosis are Acute fatty liver of pregnancy (AFLP), Viral hepatitis and Intra-hepatic cholestatis of pregnancy (ICP). These conditions also present as jaundice in pregnancy and are associated with high risk of maternal morbidity. Significant overlap in the clinical and biochemical features are noted among these conditions, nevertheless complete work-up of investigations need to be done as the treatment differs for each condition.

Jaundice and pregnancy is a lethal combination resulting in very high maternal as well as perinatal mortality and morbidity. It presents as a challenging situation to the obstetrician because of the grave complications associated with it, such as antepartum hemorrhage, post partum hemorrhage, maternal coagulopathy, intrauterine fetal demise, hepatic coma, renal failure and septicemia. There is a need to identify these cases and their obstetric outcome.

This study was thus conducted to determine the incidence, maternal and perinatal outcomes in the pregnant women who presented with jaundice.

\section{Methodology:-}

This retrospective cross-sectional study was conducted in a tertiary care centre, catering to all sections of society. Patients who presented with jaundice in pregnancy during this 5 year period (September 2011 to August 2016) were included in the study. Retrospectively, the cases who presented with jaundice in pregnancy over the past 5 years were identified by gathering data from medical records section. Individual consent was waivered off after due permission from medical records division.

All the pregnant and also post partum women referred as jaundice were included in the study. Case records with incomplete or missing data were excluded. Relevant obstetric information such as age, parity, period of gestation, history of prior liver diseases, details regarding the referral, outcome of the pregnancy, antenatal complications, mode of delivery, postnatal complications were noted. Neonatal outcome were noted in terms of neonatal complications, ICU admissions and death. Maternal ICU admissions were identified, reason for ICU admission and further management in ICU like ventilator support were collected from the case records. The maternal mortality and cause of maternal death, discharge against medical advice were noted from the case records. Cause of jaundice and maternal complications were investigated and all the above collected data was entered in the data collection instrument. Analysis was carried out for the entire dataset using SPSS software. Categorical data was expressed in terms of percentages.

\section{Results:-}

During the study period, 26,973 deliveries occurred, among which $123(0.45 \%)$ cases of jaundice in pregnancy were reported. Out of these, $109(88.6 \%)$ were diagnosed in the hospital, $14(11.3 \%)$ cases were referred from other centers with jaundice.

In the study, $17(13.8 \%)$ belonged to age group of <20years, $99(80.48 \%)$ were in $21-30 y e a r s, 6(4.8 \%)$ of them belonged to 31-35 years and $1(0.8 \%)$ was $>35$ years. A greater part of them, 73 (59.34\%) were primigravida, 30 (24.39\%) were of parity status one and $15(12.19 \%)$ and $5(4 \%)$ were of parity status 2 and 3 respectively. More than half of them, $69(56.1 \%)$ were unregistered and $54(43.9)$ cases were registered for antenatal checkup. 17 (13.8\%) cases presented with postnatal jaundice and $106(86.17 \%)$ cases were antenatal cases.

Gestational age of these 123 cases was as mentioned. 1 case presented at $<28$ weeks, 16 (13\%) between 28-32 weeks, $47(38.2 \%)$ between 33-36 weeks, $57(46.34 \%)$ and $2(1.6 \%)$ were in $37-40$ weeks and > 40 weeks 
respectively. $77(62.6 \%)$ of them had spontaneous onset of labour. $64(52.03 \%)$ cases delivered preterm and 59 $(47.96 \%)$ delivered at term. Mode of delivery in $74(60.16 \%)$ cases was vaginal and $43(34.95 \%)$ cases underwent caesarean section (graph 1). Instrumental delivery was conducted in $6(4.8 \%)$ of them.

Distribution of cause of jaundice was as follows( table 1). HELLP syndrome 49 (39.84\%), viral hepatitis 39 (31.7\%), AFLP (15.4\%), cholestatic jaundice (7.3\%) and Leptospirosis was detected in 4.06\% of patients. Gilbert syndrome was found in 2 patients. Gilbert syndrome was found in 2 patients. Among these patients, a greater part of them recovered $(69.1 \%)$ but $38(30.98 \%)$ of them needed transfer to sub specialty departments and $18(14.6 \%)$ needed ventilator support. Mortality occurred in 26 (21\%) patients (graph 2). PPH (13) and DIC (13) most common cause of mortality. 8 patients had septicemia and 2 had renal dysfunction and ARF. One was referred with mismatched blood transfusion. Pulmonary edema and hepatic coma was also reported

Graph 1:- Mode of delivery

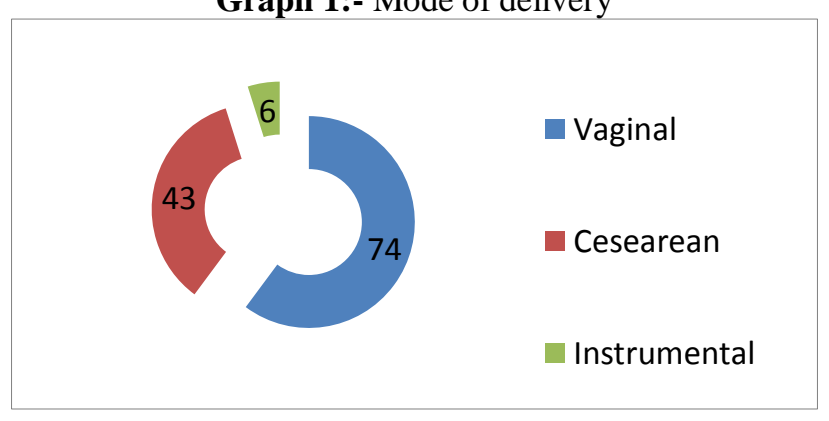

Table 1:- Distribution of cause of jaundice

\begin{tabular}{|c|c|c|}
\hline Cause of jaundice & Number & Percentage \\
\hline HELLP & 49 & $39.84 \%$ \\
\hline Viral Hepatitis & 39 & \multirow{2}{*}{31.7} \\
\hline HEV & 16 & \multirow{2}{*}{} \\
\hline HAV & 08 & \\
\cline { 2 - 2 } HbsAg & 12 & $7.3 \%$ \\
\cline { 2 - 2 } & 03 & $15.4 \%$ \\
\hline HAV+HEV & 09 & $4.06 \%$ \\
\hline Cholestatic jaundice & 19 & $1.62 \%$ \\
\hline AFLP & 05 & \\
\hline Leptospirosis & 02 & \\
\hline Gilbert syndrome & & \\
\hline
\end{tabular}

Graph 2:- Cause of mortality

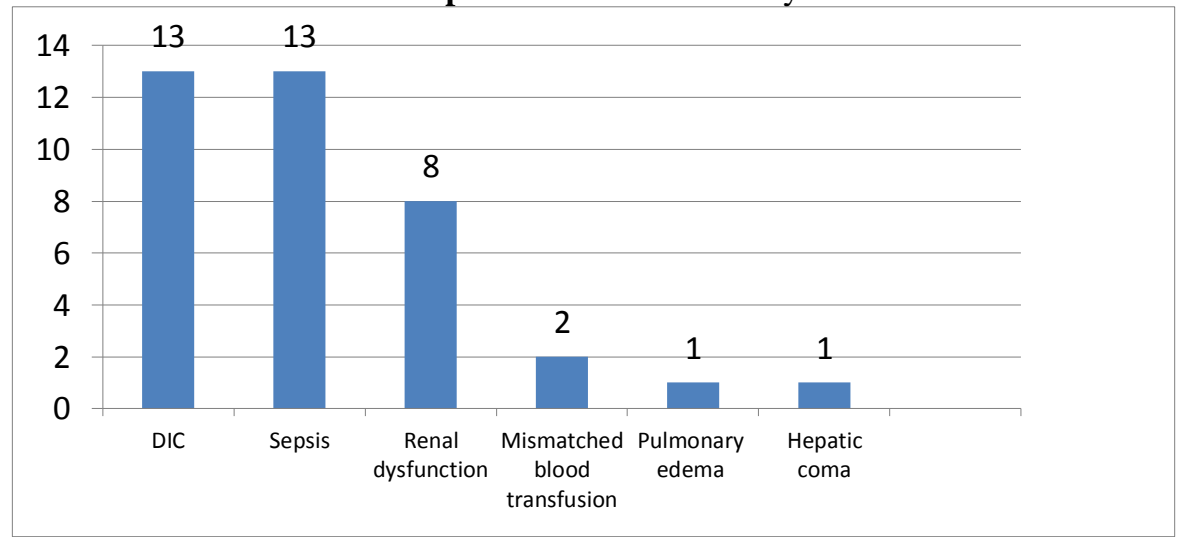

\section{Perinatal outcome:-}

Total live births were 88 ( 3 twin deliveries), 33 were still births and 5 early neonatal deaths. 66 (53.6\%) babies were females and $57(46.3 \%)$ were male babies. Majority of the babies were $2.1-2.5 \mathrm{~kg}$ at birth $48(39 \%)$. Birth weight 
distribution of babies is shown in graph 3. Requirement of NICU was seen in 59 (47.9\%) of babies with major causes for admission being preterm and low birth weight. Perinatal mortality was $30.8 \%$.

Graph 3:- Birth wt distribution of babies

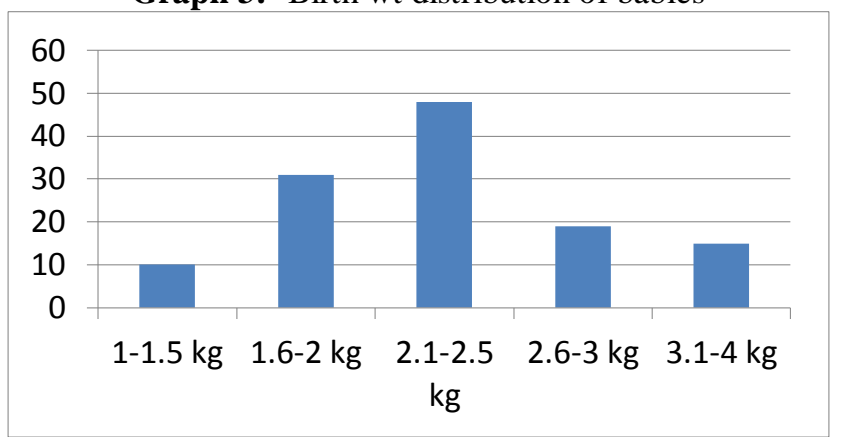

\section{Discussion:-}

The incidence of jaundice in India varies from 0.4 to $0.9 / 1000$ deliveries. ${ }^{6}$ Jaundice in pregnancy is associated with high maternal and perinatal mortality rates. In the present study, 123 women were reported to have jaundice in pregnancy among total 26,973 deliveries bringing the incidence to about $0.45 \%$. Similar results were observed in another retrospective study, which included 48 jaundice women among 7080 pregnant women (incidence $-0.65 \%$ ). ${ }^{7}$ Prevalence noted in various other studies were $0.35 \%$ and $0.4 \%$. $^{4,5}$

Majority of the women in the present study were in the age group of 21-30 years age group (99) and a fewer number in $<20$ and $>30$ years. A study done on 7280 women out of whom 26 had jaundice had similar age distribution. ${ }^{4} \mathrm{~A}$ greater part of patients were Primipara (59.34\%) and $30(24.39 \%)$ were of parity status one. More than half of them were unregistered $(56.1 \%)$ and 14 were referred postnatal.

HELLP syndrome is a multisystem disorder complicating pregnancy in third trimester. Incidence is $0.1-0.6 \%$ of all pregnancies. ${ }^{4}$ The only definitive treatment is termination of pregnancy as it is associated with grave complications such as DIC, abruptio placenta, pulmonary edema, ARF, ARDS, stroke, hepatic failure and many more. Maternal mortality reported in this condition is $1 \% .{ }^{1}$ In the present study, HELLP syndrome was seen in $39.84 \%$.

Viral hepatitis, a notorious cause exacerbated by pregnancy is seen in 1 in 700 pregnancy and can lead to fulminant hepatic failure. ${ }^{3,8}$ Fulminant hepatitis is seen in a higher percentage in third trimester with a maternal mortality ranging as high as $15-45 \%{ }^{9,10} \mathrm{HEV}$ infection is the most commonest cause of fulminant hepatitis. Viral hepatitis was seen in $31.7 \%$ in present study. These results are parallel with the Wardha study which concluded viral hepatitis as a commonest cause with HEV being the commonest. ${ }^{5}$ Similarly, HEV was found to be commonest in present study.

AFLP is a rare, life-threatening illness with an incidence of which is less than 1 in 10,000 pregnancies and a striking maternal mortality of $18 \%{ }^{1}$ Confirmation of the diagnosis is by liver biopsy which is rarely done as there are availability of serological markers and biochemical findings of AFLP. Present study had 15.4\% patients with AFLP Cholestasis in pregnancy is another rare condition with incidence of 1 in 1000 to 1 in 10000 deliveries. In contrast to AFLP, Cholestasis has an extremely good maternal outcome, however it is known for its increasing fetal morbidity and mortality. Placental insufficiency, preterm labour, fetal distress and fetal demise are the morbidities noted. ${ }^{11}$ cholestatic jaundice was seen in $7.3 \%$ of patients in the present study.

As described above, the main causes of jaundice jeopardize both maternal as well as fetal condition. Timely identification of initiation of complications brings about a drastic fall in the morbidities. Early diagnosis and identification of the cause can help in the choice of management as there are wide variations in management options in each cause.

The study reported high incidence of preterm labour as well. Main causes of maternal mortality were septicemia, DIC, renal dysfunction. Available studies also shared similar thoughts and reported coagulation failure, hepatic coma, renal failure and septicemia as causes leading to mortality among patients with jaundice in pregnancy. ${ }^{5,8}$ It 
was also noted that there was an increase in perinatal morbidity with NICU admissions of $47.9 \%$ and perinatal mortality was $30.8 \%$.

Jaundice is an important sign on clinical examination which can be easily be missed and with the above mentioned outcomes, it is clearly evident that it has a high rates of morbidity and mortality in both mother and fetus. It is regarded as one of the leading cause for maternal death due to PPH and DIC and its increased incidence in this part of Karnataka makes it an alarming affair.

\section{Conclusion:-}

Jaundice in pregnancy results in high rates of maternal as well as fetal morbidities and mortality. HELLP and viral hepatitis were found to be predominant cause. DIC is one of the most alarming complications of jaundice comprising foremost cause leading to mortality. Early detection, early diagnosis and early initiation of proper management are essential to reduce the complications.

\section{Bibliography:-}

1. R.K.Jain. Management of Jaundice in pregnancy. Medicine Update 2010; 20:470-6

2. M.S. Khuroo, S. Kamili, Aetiology and prognostic factors in acute liver failure in India, J viral Hepat., 2003, 10, 224-31

3. Sarkar CS, Giri AK, Maity TK. Jaundice in pregnancy: A clinical study. J Indian Med association. 1992 May;(5):117-8

4. Ching ling Yi, neelam Barge, A R Dalal. Study of jaundice in pregnancy in a tertiary care institute in India. Bombay hospital journal 2011;53(2): 181-3

5. Aharya Neema, Acharya Sourya, Shukla Samarth, Athvale Rutuja, Shaveta. Study of jaundice in pregnancy. Global journal of medical research Gynecology and obstetrics 2013;13(2): 25-9

6. Reddy M G, G. C. Prabhakar, Vijaya Sree. Maternal and fetal outcome in jaundice complicating Pregnancy. Journal of Dr. NTR University of Health Sciences 2014;3(4) 231-233 231

7. C.L Yi, N. Barge, A.R. Dalal, Study of Jaundice in Pregnancy in a tertiary care institute in india, Bombay Hospital J, 2011, 53, 56-9.

8. ACOG technical bulletin: Hepatitis in pregnancy International Journal of OB-GY: 42: 189, 1993.

9. M.S. Khuroo, Hepatitis E: The enterically transmitted Non-A, Non-B Hepatitis, J Gastroenterol 1991, 10, 96100.

10. M.S. Khuroo, S. Kamili, S. Jameel, Vertical transmission of Hepatitis E Virus, Lancet, 1995, 345, $1025-6$.

11. Tan LK . Obstetric cholestasis : current opinions and management.Ann Acad Med Singapore 2003; 32: 294-298 